\title{
DESIGN, SYNTHESIS, MOLECULAR DOCKING, AND EVALUATION OF CHROMONE BASED TETRAZOLE DERIVATIVES
}

\author{
ANUJA CHOPRA ${ }^{1,2}$, LAKHWINDER SINGH ${ }^{3 *}$, KAPOOR VK $^{2}$, RICHA DHINGRA ${ }^{4}$, NEELIMA DHINGRA ${ }^{4}$ \\ ${ }^{1}$ Research Scholar, IKG Punjab Technical University, Jalandhar- Kapurthala Highway, Near Pushpa Gujral Science City, Kapurthala(144601) \\ Punjab, India. ${ }^{2}$ Department of Pharmaceutical Sciences, G. H. G. Khalsa College of Pharmacy, Ludhiana (141104), Punjab, India. \\ ${ }^{3}$ Department of Applied Science, CGC College of Engineering, Mohali (140307), Punjab, India. ${ }^{4}$ Department of Pharmaceutical Sciences, \\ University Institute of Pharmaceutical Sciences, Panjab University, Chandigarh (160014), India. Email: lakhwinder.pharma@yahoo.com
}

Received: 21 November 2018, Revised and Accepted: 14 January 2019

\section{ABSTRACT}

Objectives: The objective of this research work was to design, synthesize, study the molecular docking, and evaluate the antimicrobial activity of some novel substituted 2-(Phenylamino)-3-(1H-tetrazol-5-yl)-4H-chromen-4-one derivatives (12a-h)

Methods: In the present work, 3-Formylchromones were transformed into pharmacologically active substituted 2-(Phenylamino)-3-(1H-tetrazol-5yl)-4H-chromen-4-one derivatives (12a-h) through a multistep reaction. Initially, synthesis of the substituted 4-0xo-2-(phenylamino)-4H-chromone-3carbaldehydes (9a-h) was carried out using substituted acetophenones (6a-h) as starting material and by employing an earlier reported method (1,3-dipolar cycloaddition reaction). Then, these synthesized compounds were converted into respective oximes (10a-h).The obtained oximes (10a-h) were further converted into nitriles (11a-h) which were finally subjected to concerted cycloaddition through stepwise addition of neutral or anionic azide species to furnish final substituted 2-(Phenylamino)-3-(1H-tetrazol-5-yl)-4H-chromen-4-one derivatives (12a-h). All the newly synthesized compounds (12a-h) and a reference compound (ciprofloxacin) were docked into the active site of TyrRS (PDB: 1JIK) by means of the BioPredicta module of VLife MDS. The synthesized compounds (12a-h) were also evaluated in vitro for their antibacterial (against Staphylococcus aureus, Bacillus subtilis, Pseudomonas aeruginosa, and Escherichia coli bacterial stains) and antifungal activities (against Aspergillus niger and Candida albicans fungal strains) using Zone of Inhibition method.

Results: The formation of substituted 2-(Phenylamino)-3-(1H-tetrazol-5-yl)-4H-chromen-4-one derivatives (12a-h) was confirmed through their spectral analysis, that is, ${ }^{1} \mathrm{H}-\mathrm{NMR},{ }^{13} \mathrm{C}-\mathrm{NMR}$, and Mass spectroscopy. During docking study, the recorded molecular binding interactions revealed that all the newly synthesized compounds (12a-h) interacted well with binding site of the enzyme. The synthesized compounds were also evaluated in vitro for their antibacterial (against $S$. aureus, B. subtilis, P. aeruginosa, and E. coli bacterial stains) and antifungal activities (against $A$. niger and C. albicans fungal strains). All the synthesized compounds exhibited moderate-to-potent antimicrobial activities.

Conclusions: All the synthesized compounds exhibited moderate-to-potent antimicrobial activity.

Keywords: Chromone, VilsmeierHaack, Antibacterial, Antifungal, 2-Anilino-3-formylchromones, Tetrazole, DNA gyrase

(c) 2019 The Authors. Published by Innovare Academic Sciences Pvt Ltd. This is an open access article under the CC BY license (http://creativecommons. org/licenses/by/4. 0/) DOI: http://dx.doi.org/10.22159/ajpcr.2019.v12i3.30860

\section{INTRODUCTION}

Chromones are the heterocyclic compounds demonstrating high degree of structural diversity. They constitute the largest and most varied family of organic compounds [1]. They are known to display a remarkable spectrum of pharmacological activities, including antitumor [2], anti-inflammatory [3], antibacterial [4], antifungal [5], antioxidant [6], antiHIV [7], vasodilation [8], antiviral [9] and anti-allergic [10] activities, etc.

As per the available literature reports, karangin (1) and pongaglabol (2) from plant Pongamia pinnata show antibacterial activity against Shigella dysenteriae and Staphylococcus aureus, respectively. 3-hydroxy-2-(1phenyl-3-aryl-4-pyrazolyl) chromones $(3,4)$ show antifungal activity against three phytopathogenic fungi [11], namely, Helminthosporium species, Fusarium oxysporum, and Alternaria alternata.

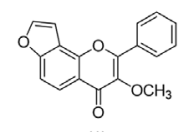

(1)

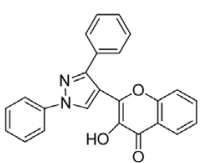

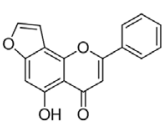

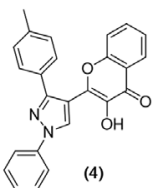

Chromones are very prone to chemical transformations such as photocycloaddition, photodimerization, photoisomerization, photorearrangement, photo-oxidation reduction, and photocyclization which involve both $n \rightarrow \pi^{*}$ and $\pi \rightarrow \pi^{*}$ transitions [12,13]. Further, chromones and bischromones (especially their 3-alkoxy derivatives) have also proven themselves as interesting substrates to study the mechanism of photochemical reactions [14].

Among their derivatives, 3-formylchromones (5) bear a unique name for being part of molecular structure of several naturally occurring and pharmacologically active heterocyclic compounds $[15,16]$.<smiles>O=c1ccoc2ccccc12</smiles>

Further, 3-Formylchromones are used as synthons in the synthesis of various heterocyclic analogs [17]. The presence of three strongly electrophilic centers (C-2 and C-4 of the chromone system as well as the carbon of the formyl group) in their structure facilitates their use in this regard and makes the chromone moiety [18] pharmacologically active. 
Despite tremendous research which has been done on chromones and 3-formylchromones, still, modern techniques are employed to synthesize their new derivatives with a focus to get improved pharmacological activities. Keeping in view the above observations, it was decided to synthesize some novel substituted 2-(Phenylamino)-3-(1H-tetrazol-5yl)-4H-chromen-4-one derivatives (12a-h, Scheme 1$)$ through reactions of various substituted 3-Formylchromones (7a-h) with different chemical reagents and evaluate them for their antimicrobial activities.

\section{MATERIALS AND METHODS}

\section{Materials}

Solvents, starting materials, and reagents were purchased from commercial suppliers and used after purification. All the solvents were purified by the standard procedure before use.

The melting points of all the synthesized compounds were measured on a liquid paraffin bath in open glass capillary tubes using Digital Melting point apparatus by Nutronics Popular Ltd. The reaction progress and product purity were checked by thin-layer chromatography using silica gel-G-coated glass plates (TLC plates) which were visualized by exposure to iodine vapors. IR spectra were recorded on Perkin-Elmer 882 model spectrometer using $\mathrm{KBr}$ pellets. Frequencies were recorded in wave number. ${ }^{1} \mathrm{H}$ NMR and ${ }^{13} \mathrm{C}$ NMR spectra were obtained on Bruker Avance II ( $300 \mathrm{MHz}$ ) NMR spectrometer for solutions in $\mathrm{CDCl}_{3} / \mathrm{DMSO}^{-\mathrm{d}_{6}}$ using tetramethylsilane as internal reference. All chemical shifts are reported in parts per million (ppm) and coupling constant (J) values in Hertz. The mass spectra were recorded on Q-TOF Micromass (liquid chromatography-mass spectrometry) instrument.

\section{Methods}

First, synthesis of the substituted 4-0xo-2-(phenylamino)-4Hchromone-3-carbaldehydes $(9 \mathrm{a}-\mathrm{h})$ was carried out starting from substituted acetophenones (6a-h) through earlier reported method [19-22]. In the next step, obtained substituted 4-0xo-2(phenylamino)-4H-chromone-3-carbaldehydes (9a-h) were again dissolved in ethanol, and hydroxylamine was added to this solution. The mixture was refluxed for $15 \mathrm{~min}$ and further stirred at room temperature for $1 \mathrm{hr}$. On completion of the reaction (monitored by TLC using hexane-ethyl acetate gradient, 9:1 v/v), the required substituted 4-0xo-2-(phenylamino)-4H-chromone-3-carbaldehyde oximes (10a-h) were obtained as solid precipitates and were purified through recrystallization using a mixture of hexane and chloroform $(9: 1 \mathrm{v} / \mathrm{v})$. Further, these 4-0xo-2-(phenylamino)-4H-chromone-3-carbaldehyde oximes (10a-h) were mixed with acetic anhydride and refluxed for $4 \mathrm{~h}$ with continuous stirring. The hot reaction mixture was then poured into crushed ice leading to the formation of precipitates of compounds 11a-h (TLC monitored, hexane: ethyl acetate [9:1 v/v]) which were then obtained by filtration [23] and further purified by recrystallization over glacial acetic acid. Finally, the obtained nitrile derivatives (11a-h) were dissolved in ice-cooled tetrahydrofuran (THF). Aluminum chloride $\left(\mathrm{AlCl}_{3}\right)$ was added to this solution. After some time, sodium azide was added to the solution. The overall reaction mixture was stirred for $10 \mathrm{~h}$, followed by cooling in ice bath for 2-3 h. Later, $5 \mathrm{ml}$ dilute $\mathrm{HCl}$ was added to it which led to the formation of crystals of final products (12a-h) which were then collected by filtration and were purified by recrystallization using hexane and chloroform (9:1 v/v, Scheme 1). The reaction conditions are summarized in Table 1 given below:

\section{Molecular docking studies}

A computational ligand-target docking approach was used to analyze structural complexes of the DNA gyrase (target) with these chromonebased heterocyclic ligands to understand the structural basis of the protein-ligand specificity $[24,25]$. All the newly synthesized compounds (12a-h) and a reference compound (ciprofloaxin) were docked [26] into

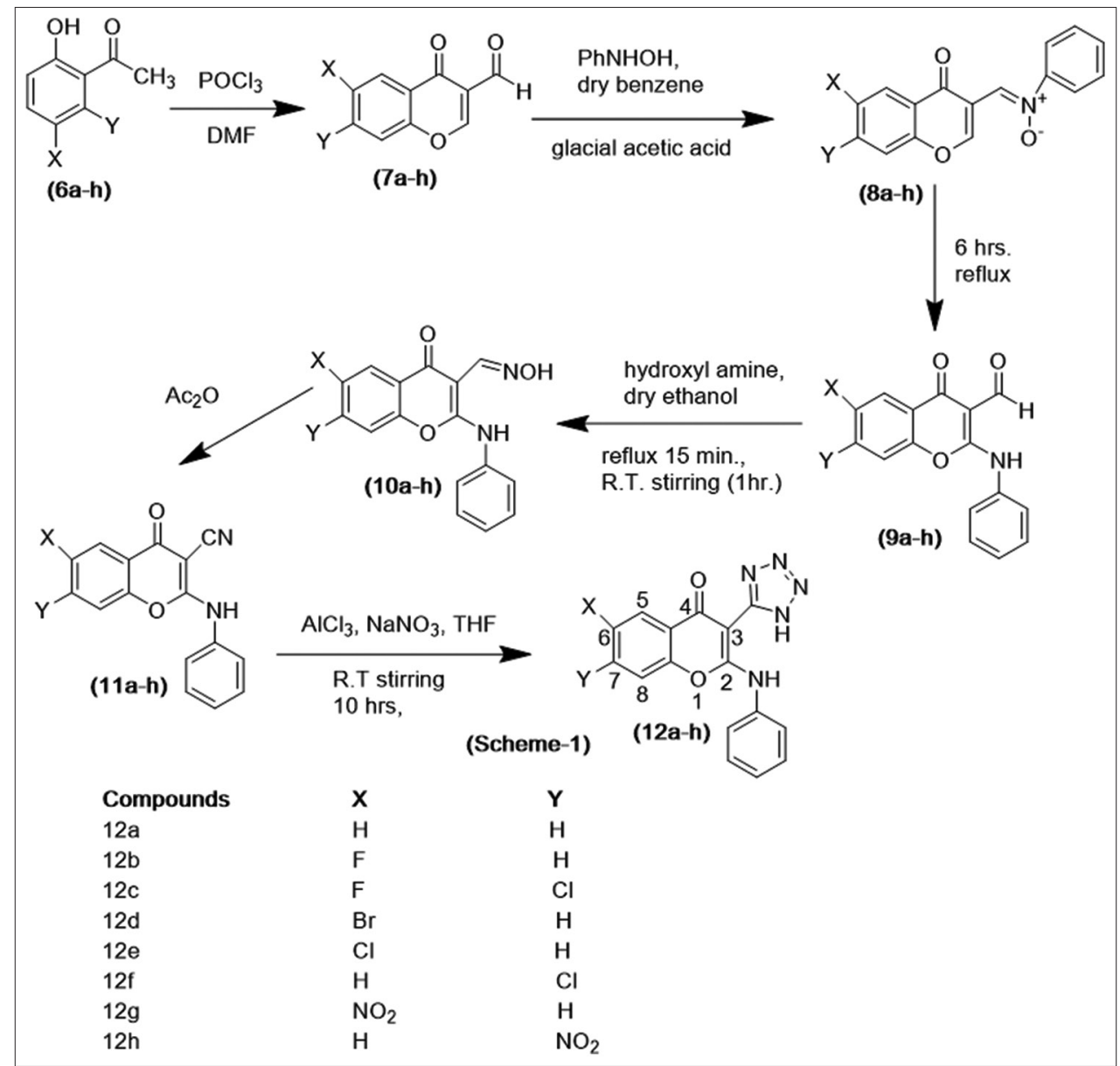

Scheme 1: Reactions conditions 
Table 1: The percentage age yield, reaction conditions are summarized in following table

\begin{tabular}{llllll}
\hline Serial number & Compound number & $\mathbf{X}$ & $\mathbf{Y}$ & Solvent & Reaction condition \\
\hline 1 & $12 \mathrm{a}$ & $\mathrm{H}$ & $\mathrm{H}$ & $\mathrm{THF}$ & Stirring RT, 10 h \\
2 & $12 \mathrm{~b}$ & $\mathrm{~F}$ & $\mathrm{H}$ & $\mathrm{THF}$ & Stirring RT, 10 h \\
3 & $12 \mathrm{c}$ & $\mathrm{F}$ & $\mathrm{Cl}$ & $\mathrm{THF}$ & Stirring RT, $10 \mathrm{~h}$ \\
4 & $12 \mathrm{~d}$ & $\mathrm{Br}$ & $\mathrm{H}$ & $\mathrm{THF}$ & Stirring RT, 10 h \\
5 & $12 \mathrm{e}$ & $\mathrm{Cl}$ & $\mathrm{H}$ & $\mathrm{THF}$ & Stirring RT, 10 h \\
6 & $12 \mathrm{f}$ & $\mathrm{H}$ & $\mathrm{Cl}$ & $\mathrm{THF}$ & Stirring RT, $10 \mathrm{~h}$ \\
7 & $12 \mathrm{~g}$ & $\mathrm{NO}_{2}$ & $\mathrm{H}$ & $\mathrm{THF}$ & Stirring RT, $10 \mathrm{~h}$ \\
8 & $12 \mathrm{~h}$ & $\mathrm{H}$ & $\mathrm{NO}_{2}$ & THF & Stirring RT, 10 h \\
\hline
\end{tabular}

THF: Tetrahydrofuran, RT: Room temperature

the active site of TyrRS (PDB: 1JIK) by means of the BioPredicta module of VLife MDS. Before interpretation and analysis of interactions, correct ligand pose assessment generally remains an important criterion for the optimal binding affinity prediction using scoring functions. Through this docking study, all the ligand poses were visually inspected. All the docked ligands were scored using the lower Dock Score function, and the pose of each ligand that matched the assumed binding mode was considered valid and put to the separate set (valid poses). The best pose of each was identified for subsequent analysis.

\section{Pharmacological activity}

The obtained compounds, that is, substituted 2-(Phenylamino)-3-(1Htetrazol-5-yl)-4H-chromen-4-one derivatives (12a-h) were evaluated for their in vitro antimicrobial activities against different strains of bacteria (S. aureus, Bacillus subtilis, Pseudomonas aeruginosa, and Escherichia coli) and fungi (Aspergillus niger and Candida albicans) as per the reported methods [13,27].

\section{RESULTS AND DISCUSSION}

The results of synthetic work have revealed that maximum yield of compounds (12a-h) was obtained in the cases where chromone nucleus was bearing electron-withdrawing groups at C-6 or C-7. All the synthesized compounds were evaluated by spectral analysis, that is, ${ }^{1} \mathrm{H}-\mathrm{NMR},{ }^{13} \mathrm{C}-\mathrm{NMR}$, and Mass spectroscopy. The spectral data of the synthesized compounds 12a-h are given below:

General procedure for the synthesis of 2-(phenylamino)-3-(1Htetrazol-5-yl)-4H-chromen-4-one (12a-h)

The obtained compounds (11a-h) were dissolved in ice-cooled THF. $\mathrm{AlCl}_{3}$ was added to this solution. After some time, sodium azide was added to the solution. The overall reaction mixture was stirred for $10 \mathrm{~h}$, followed by cooling in ice bath for 2-3 h. Later, $5 \mathrm{ml}$ dilute $\mathrm{HCl}$ was added to it which led to the formation of crystals of final products (12a-h) which were then collected by filtration and were purified by recrystallization using hexane and chloroform (9:1 v/v, Scheme 1).

2-(Phenylamino)-3-(1H-tetrazol-5-yl)-4H-chromen-4-one (12a) Reaction of 4-0xo-2-(phenylamino)-4H-chromone-3-carbonitrile (11a, $1.7 \mathrm{~g}$ ) with ice-cooled THF ( $4 \mathrm{ml}), \mathrm{AlCl}_{3}(0.5 \mathrm{~g})$, and sodium azide $(0.5 \mathrm{~g})$ was carried out, and compound 12a was obtained as brownish yellow crystals "Yield 1.02 g (60\%)," mp 183-194 ${ }^{\circ} \mathrm{C}, \mathrm{C}_{16} \mathrm{H}_{13} \mathrm{~N}_{5} \mathrm{O}_{2}$, molecular weight $305 \mathrm{~g}$, and solubility DMSO.

${ }^{1}$ H-NMR: (400 MHz, DMSO- $\left.d_{6}\right), \delta$ ppm $(J, H z): 2.45(1 \mathrm{H}, \mathrm{s}$, tetrazole); $3.73\left(1 \mathrm{H}, \mathrm{s}, \mathrm{NHC}_{6} \mathrm{H}_{5}\right) ; 7.21-6.76\left(5 \mathrm{H}, \mathrm{m}, \mathrm{C}_{6} \mathrm{H}_{5}\right) ; 7.61-7.30(3 \mathrm{Hs}, \mathrm{m}, \mathrm{Ar}-$ chromone); 7.92-7.82 (IH, dd, $J=8 \mathrm{~Hz}, \mathrm{C}_{5}$-chromone).

${ }^{13} \mathrm{C}-\mathrm{NMR}:\left(400 \mathrm{MHz}, \mathrm{DMSO}-\mathrm{d}_{6}\right), \delta, \mathrm{ppm}$

163.3 (C-2), 79.2 (C-3), 124.7 (C-4, C=0), 173.7 (C-4) 153.5 (C-8), 131.9 (C-5), 122.6 (C-6), 133.9 (C-7), 117.0 (C-8), 75.9 (5' tetrazole), 135.5 (C-1"), 116.1 (C-2",6"), 129.6 (C-3",5"), and 118.9 (C-4").

\section{Mass: $M^{+} m / z$}

$305\left(\mathrm{M}+\mathrm{C}_{16} \mathrm{H}_{11} \mathrm{~N}_{5} \mathrm{O}_{2}\right) ; 238\left(\mathrm{M}+\mathrm{C}_{15} \mathrm{H}_{11} \mathrm{NO}_{2}\right) ; 210\left(\mathrm{M}+\mathrm{C}_{13} \mathrm{H}_{11} \mathrm{ON}\right) ; 128$ $\left(\mathrm{M}+\mathrm{C}_{8} \mathrm{H}_{6} \mathrm{O}\right)$; and $94\left(\mathrm{M}+\mathrm{C}_{6} \mathrm{H}_{7} \mathrm{~N}\right)$.
6-Fluoro-2-(phenylamino)-3-(1H-tetrazol-5-yl)-4H-chromen-4one (12b)

Reaction of 6-Flouro-2-(phenylamino)-4H-chromone-3-carbonitrile $(11 \mathrm{~b}, 0.900 \mathrm{~g})$ with ice-cooled THF $(4 \mathrm{ml}), \mathrm{AlCl}_{3}(0.5 \mathrm{~g})$, and sodium azide $(0.5 \mathrm{~g})$ was carried out, and compound $12 \mathrm{~b}$ was obtained as brownish yellow crystals "Yield $0.774 \mathrm{~g}(85 \%)$," mp $229-240^{\circ} \mathrm{C}, \mathrm{C}_{16} \mathrm{H}_{12} \mathrm{O}_{2} \mathrm{~N}_{5} \mathrm{~F}$, molecular weight $323 \mathrm{~g}$, and solubility DMSO.

${ }^{1} \mathrm{H}-\mathrm{NMR}:\left(400 \mathrm{MHz}, \mathrm{DMSO}-\mathrm{d}_{6}\right), \delta, \mathrm{ppm}(\mathrm{J}, \mathrm{Hz})$

$2.52(1 \mathrm{H}, \mathrm{s}$, tetrazole $) ; 3.65\left(1 \mathrm{H}, \mathrm{s}, \mathrm{NHC}_{6} \mathrm{H}_{5}\right) ; 7.43-7.15(5 \mathrm{H}, \mathrm{m}$, $\mathrm{C}_{6} \mathrm{H}_{5}$ ); 7.84-7.54 (3H, m Ar-chromone); and 7.84-7.82 (1H, J=8 Hz, d, $\mathrm{C}_{5}$-chromone).

${ }^{13} \mathrm{C}-\mathrm{NMR}:\left(400 \mathrm{MHz}, \mathrm{DMSO}-\mathrm{d}_{6}\right), \delta, \mathrm{ppm}$

164.3 (C-2); 78.4 (C-3); 124.7 (C-4, C=0); 125.6 (C-4);152.7 (C-8a); 115.1 (C-5); 171.1 (C-6); 122.0 (C-7); 118.3 (C-8); 79.1 (5' tetrazole); 135.5 (C-1"); 116.2 (C-2",6"); 129.5 (C-3",5"); and 118.8 (C-4").

Mass: $M^{+} m / z$

$323\left(\mathrm{M}+\mathrm{C}_{16} \mathrm{H}_{10} \mathrm{O}_{2} \mathrm{~N}_{5} \mathrm{~F}\right)$ and $304\left(\mathrm{M}+\mathrm{C}_{16} \mathrm{H}_{10} \mathrm{O}_{2} \mathrm{~N}_{5}\right)(100)$.

6-Fluoro-7-chloro-2-(phenylamino)-3-(1H-tetrazol-5-yl)-4Hchromen-4-one (12c)

Reaction of 6-Flouro-7-chloro-2-(phenylamino)-4H-chromone-3carbonitrile (11c, $0.721 \mathrm{~g}$ ) with ice-cooled THF ( $4 \mathrm{ml}), \mathrm{AlCl}_{3}(0.5 \mathrm{~g})$, and sodium azide $(0.5 \mathrm{~g})$ was carried out, and compound $12 \mathrm{c}$ was obtained as brownish yellow crystals "Yield $0.556 \mathrm{~g}$ (72)," $\mathrm{mp} 233-245^{\circ} \mathrm{C}$, $\mathrm{C}_{16} \mathrm{H}_{11} \mathrm{O}_{2} \mathrm{~N}_{5} \mathrm{FCl}$, molecular weight $357 \mathrm{~g}$, and solubility DMSO.

${ }^{1} \mathrm{H}-\mathrm{NMR}:\left(400 \mathrm{MHz}, \mathrm{DMSO}-\mathrm{d}_{6}\right), \delta, \mathrm{ppm}(\mathrm{J}, \mathrm{Hz})$

$2.53\left(1 \mathrm{H}, \mathrm{s}\right.$, tetrazole); $3.36\left(1 \mathrm{H}, \mathrm{s}, \mathrm{NHC}_{6} \mathrm{H}_{5}\right) ; 7.62-7.16\left(5 \mathrm{H}, \mathrm{m}, \mathrm{C}_{6} \mathrm{H}_{5}\right)$; 8.03-8.01 (1H, d, J=8 Hz, C 8 -chromone); and 8.27-8.25 (IH, J=4 Hz, t, $\mathrm{C}_{5}$ chromone).

${ }^{13} \mathrm{C}-\mathrm{NMR}:\left(400 \mathrm{MHz}, \mathrm{DMSO}-\mathrm{d}_{6}\right), \delta, \mathrm{ppm}$

163.1 (C-2); 79.2 (C-3); 124.2 (C-4, C=0); 122.5 (C-4 $)$; 138.1 (C-8); 128.7 (C-5); 124.6 (C-6); 129.4 (C-7); 120.2 (C-8); 78.6 (5' tetrazole); 135.2 (C-1"); 116.0 (C-2",6"); 129.1 (C-3",5"); and 118.5 (C-4").

Mass: $M^{+} m / z$

$357\left(\mathrm{M}+\mathrm{C}_{16} \mathrm{H}_{9} \mathrm{O}_{2} \mathrm{~N}_{5} \mathrm{FCl}\right) ; 313\left(\mathrm{M}+\mathrm{C}_{15} \mathrm{H}_{\mathrm{i0}} \mathrm{N}_{5} \mathrm{OCl}\right) ; 198\left(\mathrm{M}+\mathrm{C}_{10} \mathrm{H}_{5} \mathrm{O}_{2} \mathrm{FCl}\right)$; and $254\left(\mathrm{M}+\mathrm{C}_{9} \mathrm{H}_{5} \mathrm{ON} \mathrm{N}_{5} \mathrm{FCl}\right)$.

6-Bromo-2-(phenylamino)-3-(1H-tetrazol-5-yl)-4H-chromen-4one (12d)

Reaction of 6-Bromo-2-(phenylamino)-4H-chromone-3-carbonitrile (11d, $0.946 \mathrm{~g}$ ) with ice-cooled THF (4 ml), $\mathrm{AlCl}_{3}(0.5 \mathrm{~g})$, and sodium azide $(0.5 \mathrm{~g})$ was carried out, and compound $12 \mathrm{~d}$ was obtained as brownish yellow crystals "Yield 0.651 g (69\%)," mp 208-220 ${ }^{\circ} \mathrm{C}$, $\mathrm{C}_{16} \mathrm{H}_{12} \mathrm{O}_{2} \mathrm{~N}_{5} \mathrm{Br}$, molecular weight $384 \mathrm{~g}$, and solubility DMSO. ${ }^{1} \mathrm{H}-\mathrm{NMR}$ : $\left(400 \mathrm{MHz}, \mathrm{DMSO}-d_{6}\right), \delta \mathrm{ppm}(\mathrm{J}, \mathrm{Hz}): 2.40(1 \mathrm{H}, \mathrm{s}$, tetrazole); $3.38(1 \mathrm{H}, \mathrm{s}$, $\mathrm{NHC}_{6} \mathrm{H}_{5}$ ); 7.24-6.75 (5H, m, $\left.\mathrm{C}_{6} \mathrm{H}_{5}\right)$; 7.54-7.40 (3H, m, Ar-chromone); and 7.82-7.80 (1H, J=4 Hz, d, $\mathrm{C}_{5}$-chromone). 
${ }^{13} \mathrm{C}-\mathrm{NMR}:\left(400 \mathrm{MHz}, \mathrm{DMSO}-\mathrm{d}_{6}\right), \delta, \mathrm{ppm}$

163.1 (C-2); 77.4 (C-3); 124.1 (C-4, C=0); 126.1 (C-4); 169.1 (C-8); 133.9 (C-5); 117.0 (C-6); 135.5 (C-7); 118.9 (C-8); 76.7 (5' tetrazole); 135.2 (C-1"); 116.2 (C-2",6"); 129.2 (C-3",5"); and 118.4 (C-4").

Mass

Found, $m / z: 384\left(\mathrm{M}^{+}\right) \cdot\left(\mathrm{C}_{16} \mathrm{H}_{10} \mathrm{O}_{2} \mathrm{~N}_{5} \mathrm{Br}\right)$. Calculated, $m / z: 383.9$.

6-Chloro-2-(phenylamino)-3-(1H-tetrazol-5-yl)-4H-chromen-4one (12e)

Reaction of 6-Chloro-2-(phenylamino)-4H-chromone-3-carbonitrile $(11 \mathrm{e}, 0.971 \mathrm{~g})$ with ice-cooled THF ( $4 \mathrm{ml}), \mathrm{AlCl}_{3}(0.5 \mathrm{~g})$, and sodium azide ( $0.5 \mathrm{~g}$ ) was carried out, and compound $12 \mathrm{e}$ was obtained as brownish yellow crystals "Yield 0.623 g (64)," mp $187-200^{\circ} \mathrm{C}, \mathrm{C}_{16} \mathrm{H}_{12} \mathrm{~N}_{5} \mathrm{O}_{2} \mathrm{Cl}$, molecular weight $339 \mathrm{~g}$, and solubility DMSO.

${ }^{1} \mathrm{H}-\mathrm{NMR}:\left(400 \mathrm{MHz}, \mathrm{DMSO}-\mathrm{d}_{6}\right), \delta, \mathrm{ppm}(\mathrm{J}, \mathrm{Hz})$

$2.43(1 \mathrm{H}, \mathrm{s}$, tetrazole $) ; 4.01\left(1 \mathrm{H}, \mathrm{s}, \mathrm{NHC}_{6} \mathrm{H}_{5}\right) ; 7.63-7.08\left(5 \mathrm{H}, \mathrm{m}, \mathrm{C}_{6} \mathrm{H}_{5}\right) ; 8.02-$ 7.64 (3H, m, Ar-chromone); and 8.25-8.21 ( $\mathrm{IH}, J=8 \mathrm{~Hz}, \mathrm{~d}, \mathrm{C}_{5}$-chromone).

${ }^{13} \mathrm{C}$-NMR: $\left(400 \mathrm{MHz}, \mathrm{DMSO}-\mathrm{d}_{6}\right), \delta, \mathrm{ppm}$

163.0 (C-2); 78.6 (C-3); 124.3 (C-4, C=0); 125.7 (C-4a); 153.5 (C-8); 129.6 (C-5); 129.2 (C-6); 135.3 (C-7); 118.4 (C-8); 78.0 (5' tetrazole); 135.2 (C-1"); 116.4 (C-2",6"); 129.4 (C-3",5"); and 118.8 (C-4").

\section{Mass: $M^{+} m / z$}

$339\left(\mathrm{M}+\mathrm{C}_{16} \mathrm{H}_{10} \mathrm{~N}_{5} \mathrm{O}_{2} \mathrm{Cl}\right) ; 311\left(\mathrm{M}+\mathrm{C}_{15} \mathrm{H}_{10} \mathrm{~N}_{5} \mathrm{OCl}\right) ; 237\left(\mathrm{M}+\mathrm{C}_{15} \mathrm{H}_{11} \mathrm{NO}_{2}\right)$; and $179\left(\mathrm{M}+\mathrm{C}_{9} \mathrm{H}_{5} \mathrm{ClO}_{2}\right)$

\section{7-Chloro-2-(phenylamino)-3-(1H-tetrazol-5-yl)-4H-chromen-4-} one (12f)

Reaction of 7-Chloro-2-(phenylamino)-4H-chromone-3-carbonitrile (11f, $0.643 \mathrm{~g}$ ) with ice-cooled THF ( $4 \mathrm{ml}), \mathrm{AlCl}_{3}(0.5 \mathrm{~g})$, and sodium azide $(0.5 \mathrm{~g})$ was carried out, and compound $12 \mathrm{f}$ was obtained as brownish yellow crystals "Yield $0.589 \mathrm{~g}(92)^{\prime}$ ", mp192-204 ${ }^{\circ} \mathrm{C}, \mathrm{C}_{16} \mathrm{H}_{12} \mathrm{~N}_{5} \mathrm{O}_{2} \mathrm{Cl}$, molecular weight $339 \mathrm{~g}$, and solubility DMSO.

${ }^{1} \mathrm{H}-\mathrm{NMR}:\left(400 \mathrm{MHz}, \mathrm{DMSO}-\mathrm{d}_{6}\right), \delta, \mathrm{ppm}(\mathrm{J}, \mathrm{Hz})$

$1.89\left(1 \mathrm{H}, \mathrm{s}\right.$, tetrazole); $3.37\left(1 \mathrm{H}, \mathrm{s}, \mathrm{NHC}_{6} \mathrm{H}_{5}\right) ; 7.63-7.15\left(5 \mathrm{H}, \mathrm{m}^{\mathrm{C}} \mathrm{C}_{6} \mathrm{H}_{5}\right) ; 7.63-$ 7.60 (3H, m, Ar-chromone); and 8.01-7.90 ( $\mathrm{IH}, \mathrm{J}=9.6 \mathrm{~Hz}, \mathrm{~d}, \mathrm{C}_{5}$-chromone).

${ }^{13} \mathrm{C}-\mathrm{NMR}:\left(400 \mathrm{MHz}, \mathrm{DMSO}-\mathrm{d}_{6}\right), \delta, \mathrm{ppm}$

163.1 (C-2); 79.2 (C-3); 124.2 (C-4, C=0); 122.5 (C-4 ); 138.1 (C-8); 128.7 (C-5); 124.6 (C-6); 129.4 (C-7); 120.2 (C-8); 78.6 (5' tetrazole); 135.2 (C-1"); 116.0 (C-2",6"); 129.1 (C-3",5"); and 118.5 (C-4").

\section{Mass}

Found, $m / z: 339\left(\mathrm{M}^{+}\right) \cdot\left(\mathrm{C}_{16} \mathrm{H}_{10} \mathrm{~N}_{5} \mathrm{O}_{2} \mathrm{Cl}\right)$. Calculated, $m / z: 339.5$.

\section{6-Nitro-2-(phenylamino) 3-(1H-tetrazol-5-yl)-4H-chromen-4-one} (12g)

Reaction of 6-Nitro-2-(phenylamino)-4H-chromone-3-carbonitrile $(11 \mathrm{~g}, 0.920 \mathrm{~g})$ with ice-cooled THF (4 ml), $\mathrm{AlCl}_{3}(0.5 \mathrm{~g})$, and sodium azide $(0.5 \mathrm{~g})$ was carried out, and compound $12 \mathrm{~g}$ was obtained as brownish yellow crystals "Yield 0.572 g (62)," mp $179^{\circ} \mathrm{C}-192^{\circ} \mathrm{C}$, $\mathrm{C}_{16} \mathrm{H}_{12} \mathrm{~N}_{6} \mathrm{O}_{4}$, molecular weight $350 \mathrm{~g}$, and solubility DMSO.

${ }^{1} \mathrm{H}-\mathrm{NMR}:\left(400 \mathrm{MHz}, \mathrm{DMSO}-\mathrm{d}_{6}\right), \delta, \mathrm{ppm}(\mathrm{J}, \mathrm{Hz})$

$2.51\left(1 \mathrm{H}, \mathrm{s}\right.$, tetrazole); $3.34\left(1 \mathrm{H}, \mathrm{s}, \mathrm{NHC}_{6} \mathrm{H}_{5}\right) ; 7.53-7.17\left(5 \mathrm{H}, \mathrm{m}, \mathrm{C}_{6} \mathrm{H}_{5}\right) ; 8.2-$ 8.0 ( $3 \mathrm{H}, \mathrm{M}, \mathrm{Ar}$-chromone); and 8.32-8.20 ( $\mathrm{IH}, \mathrm{J}=2.6 \mathrm{~Hz}, \mathrm{~d}, \mathrm{C}_{5}$-chromone).

${ }^{13} \mathrm{C}-\mathrm{NMR}:\left(400 \mathrm{MHz}, \mathrm{DMSO}-\mathrm{d}_{6}\right), \delta, \mathrm{ppm}$

163.0 (C-2); 78.9 (C-3); 124.3 (C-4, C=0); 124.7 (C-4); 125.7 (C-8); 131.9 (C-5); 152.3 (C-6); 127.8 (C-7); 118.9 (C-8); 78.3 (5' tetrazole); 135.3 (C-1"); 116.3 (C-2",6"); 129.3 (C-3",5"); and 117.0 (C-4").
Mass: $M^{+} m / z$

$350\left(\mathrm{M}+\mathrm{C}_{16} \mathrm{H}_{10} \mathrm{~N}_{6} \mathrm{O}_{4}\right) ; 322\left(\mathrm{M}+\mathrm{C}_{15} \mathrm{H}_{10} \mathrm{~N}_{6} \mathrm{O}_{3}\right) ; 280\left(\mathrm{M}+\mathrm{C}_{15} \mathrm{H}_{10} \mathrm{~N}_{2} \mathrm{O}_{4}\right) ; 254$ $\left(\mathrm{M}+\mathrm{C}_{9} \mathrm{H}_{6} \mathrm{~N}_{6} \mathrm{O}_{3}\right) ; 230\left(\mathrm{M}+\mathrm{C}_{10} \mathrm{H}_{7} \mathrm{~N}_{5} \mathrm{O}_{2}\right) ;$ and $206\left(\mathrm{M}+\mathrm{C}_{9} \mathrm{H}_{6} \mathrm{~N}_{2} \mathrm{O}_{4}\right)$.

7-Nitro-2-(phenylamino)-3-(1H-tetrazol-5-yl)-4H-chromen-4-one (12h)

Reaction of 7-Nitro-2-(phenylamino)-4H-chromone-3-carbonitrile $(11 \mathrm{~h}, 0.882 \mathrm{~g})$ with ice-cooled THF $(4 \mathrm{ml}), \mathrm{AlCl}_{3}(0.5 \mathrm{~g})$, and sodium azide $(0.5 \mathrm{~g})$ was carried out, and compound $12 \mathrm{~h}$ was obtained as brownish yellow crystals “Yield $0.723 \mathrm{~g}(82)^{\prime}$ ”, $\mathrm{mp} 185-198^{\circ} \mathrm{C}, \mathrm{C}_{16} \mathrm{H}_{12} \mathrm{~N}_{6} \mathrm{O}_{4^{\prime}}$ molecular weight $350 \mathrm{~g}$, and solubility DMSO.

${ }^{1} \mathrm{H}-\mathrm{NMR}:\left(400 \mathrm{MHz}, \mathrm{DMSO}-\mathrm{d}_{6}\right), \delta, \mathrm{ppm}(\mathrm{J}, \mathrm{Hz})$

$2.6\left(1 \mathrm{H}, \mathrm{s}\right.$, tetrazole); $3.36\left(1 \mathrm{H}, \mathrm{s}, \mathrm{NHC}_{6} \mathrm{H}_{5}\right) ; 7.52-7.18\left(5 \mathrm{H}, \mathrm{m}, \mathrm{C}_{6} \mathrm{H}_{5}\right)$; 8.02-7.80 (3H, m, Ar-chromone); and 8.25-8.21 $(1 \mathrm{H}, J=2.6 \mathrm{~Hz}, \mathrm{~d}$, $\mathrm{C}_{5}$-chromone)

${ }^{13} \mathrm{C}-\mathrm{NMR}:\left(400 \mathrm{MHz}, \mathrm{DMSO}-\mathrm{d}_{6}\right), \delta, \mathrm{ppm}$

163.2 (C-2); 79.0 (C-3); 124.6 (C-4, C=0); 132.8 (C-4); 153.5 (C-8); 134.1 (C-5); 116.1 (C-6); 152.3 (C-7); 114.5 (C-8); 78.3 (5' tetrazole); 135.3 (C-1"); 116.4 (C-2",6"); 129.7 (C-3",5"); and 118.7 (C-4").

Mass: $M^{+} m / z$

$350\left(\mathrm{M}+\mathrm{C}_{16} \mathrm{H}_{10} \mathrm{~N}_{6} \mathrm{O}_{4}\right)$ and $254\left(\mathrm{M}+\mathrm{C}_{9} \mathrm{H}_{6} \mathrm{~N}_{6} \mathrm{O}_{3}\right)$.

\section{Molecular docking analysis}

The results of the docking simulation study represented as D-Score are shown in Table 2. The recorded binding interactions revealed that all the newly synthesized compounds interacted well with binding site of enzyme. Further, it was also observed that the number of the substituent groups and their respective positions on the aryl moiety affects the orientation and binding pattern of the compounds in the binding pocket of the receptor. Based on the experimental results of in vitro and in vivo investigations, the detailed interaction analysis was performed on selected compound, that is, 12a, 12b, and 12c. Most stable conformers of 12a, 12b, and 12c (namely, LP-2, LP-4, and LP-5) afforded $-69.674,-72.682$, and -64.547 D score values, respectively, as compared to the reference compound, ciprofloxacin, that exhibited D score value of 43.93 against TyrRS (PDB: 1JIK). Table 3 presents the potential interactions such as hydrogen bonding, aromatic interactions, Van der Waal, and hydrophobic ones between the protein and the synthesized compounds $12 \mathrm{a}, 12 \mathrm{~b}, 12 \mathrm{c}$, and ciprofloxacin, respectively.

In compound 12a, (2-(Phenylamino)-3-(1H-tetrazol-5-yl)-4Hchromen-4-one) which is an unsubstituted derivative, chromen-4one group was found to interact through hydrogen bonding with GLN 196A amino acid and through aromatic interactions with HIS 50A amino acid, assuring a bond distance of 1.74 and $3.95 \AA$, respectively, whereas, in 6-Fluoro-2-(phenylamino)-3-(1H-tetrazol-5-yl)- $4 H$ chromen-4-one $(12 \mathrm{~b})$ that has a fluoro-substitution, $\mathrm{C}=\mathrm{O}$ group of chromen-4-one group was found involved in hydrogen bonding with GLN 196A having force distance of $1.77 \AA$, along with the hydrogen bonding between its fluoro group and GLN 193A amino acid at a force distance of $1.77 \AA$, as shown in Fig.1. Aromatic interactions were also

Table 2: D score of synthesized compounds (12a-h)

\begin{tabular}{lllll}
\hline Serial number & Compound number & $\mathbf{X}$ & $\mathbf{Y}$ & D-scores \\
\hline 1 & $12 \mathrm{a}$ & $\mathrm{H}$ & $\mathrm{H}$ & -69.674574 \\
2 & $12 \mathrm{~b}$ & $\mathrm{~F}$ & $\mathrm{H}$ & -72.682812 \\
3 & $12 \mathrm{c}$ & $\mathrm{F}$ & $\mathrm{Cl}$ & -68.884069 \\
4 & $12 \mathrm{~d}$ & $\mathrm{Br}$ & $\mathrm{H}$ & -64.547849 \\
5 & $12 \mathrm{e}$ & $\mathrm{Cl}$ & $\mathrm{H}$ & -70.176320 \\
6 & $12 \mathrm{f}$ & $\mathrm{H}$ & $\mathrm{Cl}$ & -67.163534 \\
7 & $12 \mathrm{~g}$ & $\mathrm{NO}_{2}$ & $\mathrm{H}$ & -67.722374 \\
8 & $12 \mathrm{~h}$ & $\mathrm{H}$ & $\mathrm{NO}_{2}$ & -71.268687 \\
Standard drug & Ciprofloxacin & & & -43.934875 \\
\hline
\end{tabular}


Table 3: Indicates the potential interactions

\begin{tabular}{|c|c|c|c|c|c|c|c|c|c|}
\hline Serial number & Compound number & $\mathbf{X}$ & $\mathbf{Y}$ & Ligand pose & D-score & Residues & Hydrogen & Hydro phobic & Aromatic \\
\hline 1 & $12 \mathrm{a}$ & $\mathrm{H}$ & $\mathrm{H}$ & LP2 & -69.674574 & 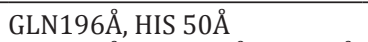 & + & - & + \\
\hline 2 & $12 \mathrm{~b}$ & $\mathrm{~F}$ & $\mathrm{H}$ & LP4 & -72.682812 & 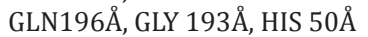 & ++ & - & + \\
\hline 3 & $12 \mathrm{c}$ & $\mathrm{F}$ & $\mathrm{Cl}$ & LP3 & -68.884069 & 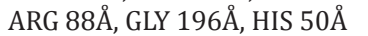 & ++ & - & + \\
\hline 4 & Ciprofloxacin & & & LP1 & -43.934875 & 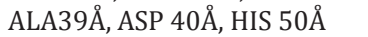 & - & +++ & _ \\
\hline
\end{tabular}

Table 4: Antibacterial results of all compounds (12a-h) against Staphylococcus aureus, Escherichia coli, Bacillus subtilis, and Pseudomonas aeruginosa by Kirby-Bauer disc diffusion method

\begin{tabular}{|c|c|c|c|c|}
\hline \multirow[t]{3}{*}{ Synthesized compounds } & \multicolumn{4}{|l|}{ Antibacterial activity } \\
\hline & \multicolumn{4}{|c|}{ Minimum inhibitory concentration $(\mu \mathrm{g} / \mathrm{mL})$} \\
\hline & Staphylococcus aureus & Escherichia coli & Bacillus subtilis & Pseudomonas aeruginosa \\
\hline $12 \mathrm{a}$ & 30 & 30 & 30 & 30 \\
\hline $12 \mathrm{~b}$ & 30 & 30 & 30 & 30 \\
\hline $12 \mathrm{c}$ & 30 & 30 & 50 & 30 \\
\hline $12 \mathrm{~d}$ & 50 & 100 & 50 & 50 \\
\hline $12 \mathrm{f}$ & 30 & 50 & 30 & 100 \\
\hline $12 \mathrm{~g}$ & 30 & 100 & 30 & 30 \\
\hline $12 \mathrm{~h}$ & 50 & 100 & 50 & 50 \\
\hline Ciprofloxacin (standard) & 30 & 30 & 30 & 30 \\
\hline
\end{tabular}

Table 5: Antifungal results of compounds 12a-h against Candida albicans and Aspergillus niger by Kirby-Bauer disc diffusion method

\begin{tabular}{lll}
\hline Compounds & \multicolumn{2}{l}{ Antifungal activity } \\
\cline { 2 - 3 } & $\begin{array}{l}\text { Minimum inhibitory } \\
\text { concentration }(\boldsymbol{\mu g} / \mathbf{m L})\end{array}$ \\
\cline { 2 - 3 } & \multicolumn{1}{l}{ Candida albicans } & Aspergillus niger \\
\hline $12 \mathrm{a}$ & 30 & 50 \\
$12 \mathrm{~b}$ & 30 & 30 \\
$12 \mathrm{c}$ & 50 & 30 \\
$12 \mathrm{~d}$ & 30 & 100 \\
$12 \mathrm{e}$ & 30 & 100 \\
$12 \mathrm{f}$ & 50 & 50 \\
$12 \mathrm{~g}$ & 50 & 30 \\
$12 \mathrm{~h}$ & 30 & 100 \\
Fluconazole (standard) & 30 & 30 \\
\hline
\end{tabular}

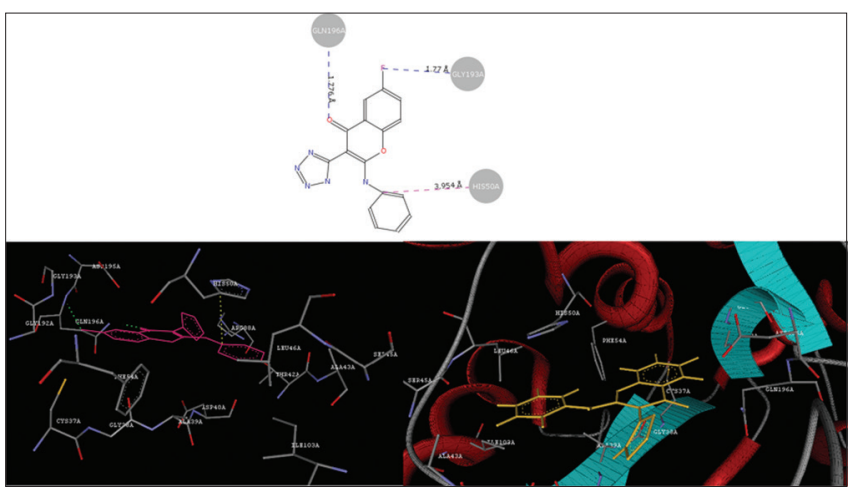

Fig. 1: Two-dimensional and three-dimensional representation of the compound $12 \mathrm{~b}$ docked in pocket site of DNA gyrase, indicating different interactions involved with amino residue

observed in its binding with residue HIS 50A, at the bond distance of $3.95 \AA$. Further, the highest $\mathrm{D}$ score of the $12 \mathrm{~b}$ is in agreement with its in vitro antibacterial and antifungal activity results. Similarly, $12 c$, the disubstituted Halo derivative of $4 \mathrm{H}$-chromen-4-one nucleus, was found to interact with residue Arg $88 \mathrm{~A}$ through hydrogen bonding involving $\mathrm{N}$-atom of the tetrazole ring (force distance of $1.56 \AA$ ) and with Gln $196 \mathrm{~A}$ residue through $\mathrm{C}=0$ group of chromen-4-one nucleus. Aromatic interactions have also been observed between the residues HIS 50A and 2-(phenylamino) of chromen-4-one nucleus, having a bond distance of $3.91 \AA$.

\section{Pharmacological activity}

Results of their antibacterial and antifungal activities [28] of synthesized compounds (12a-h) are shown in Tables 4 and 5, respectively. Most of the compounds exhibited potent antibacterial as well as antifungal activity against all the microbes tested as compared to the standard drugs used (i.e., ciprofloxacin and fluconazole for bacterial and fungal strains, respectively).

The results revealed (Table 4) that compounds $12 \mathrm{a}, 12 \mathrm{~b}$, and $12 \mathrm{e}$ presented themselves as more effective against both Gram-positive and Gram-negative bacteria. However, compounds 12d, 12f, 12g, and $12 \mathrm{~h}$ emerged as more effective against Gram-positive bacteria. Compound $12 \mathrm{c}$ was found to be more effective only against Gramnegative bacteria. It has been concluded from Kirby-Bauer disc diffusion method that the most active compounds, that is, $12 \mathrm{a}, 12 \mathrm{~b}$, and $12 \mathrm{e}$ are the broad-spectrum candidates, and other lesser active drugs, that is, $12 \mathrm{c}, 12 \mathrm{~d}, 12 \mathrm{f}, 12 \mathrm{~g}$, and $12 \mathrm{~h}$ are narrow-spectrum analogs.

The results showed (Table 5) that compounds $12 \mathrm{~b}$ and $12 \mathrm{f}$ were effective against both $C$. albicans and A. niger. Compounds 12a, 12d, $12 \mathrm{e}$, and $12 \mathrm{~h}$ were more effective against $C$. albicans only. Similarly, compounds $12 \mathrm{c}$ and $12 \mathrm{~g}$ exhibited more potent activity against $A$. niger only. It has been concluded from Kirby-Bauer disc diffusion method that the most active drug, that is, $12 \mathrm{~b}$ is broad spectrum in nature and the lesser active derivatives, that is, 12a, 12c, 12d, 12e, 12f, 12g, and $12 \mathrm{~h}$ are narrow-spectrum contenders.

\section{CONCLUSIONS}

Novel chromone-based tetrazole derivatives (12a-h) were designed, synthesized, docked, and evaluated for their in vitro antibacterial and antifungal activities against various bacterial (Gram-positive and Gram-negative) and fungal strains, respectively. 
The docking studies thus revealed that derivatives with electron-withdrawing groups play a critical role in drug-receptor interactions as exemplified by the flouro derivative with good D score. (6-Fluoro-2-(phenylamino)-3-(1H-tetrazol-5-yl)- $4 H$ chromen-4-one), $12 \mathrm{~b}$ (displayed good number of interaction at lesser bond distance with receptor as compared to the 12a), and 12c molecule, respectively, indicating $12 \mathrm{~b}$ molecule binds more strongly with the receptor with lesser distance as compared to another one. The docking studies predicted almost the same behavior as was observed in in vitro and in vivo biological evaluations for the different substituted groups among the derivatives. Overall, a good correlation was observed between the docking study and biological evaluation of active compound.

The obtained compounds substituted 2-(Phenylamino)-3-(1Htetrazol-5-yl)-4H-chromen-4-one derivatives (12a-h) were found to be potent against different bacterial strains (S. aureus, B. subtilis, P. aeruginosa, and E. coli) and fungal strains (A. niger and C. albicans) when compared with standard drug ciprofloxacin for bacterial strains and fluconazole for fungal strains. The compounds $12 \mathrm{a}, 12 \mathrm{~b}$, and 12 e were active against $S$. aureus, B. subtilis, P. aeruginosa, and E. coli, respectively, at minimum inhibitory concentration (MIC) $30 \mu \mathrm{g} / \mathrm{ml}$ when compared with standard drug ciprofloxacin and rest compounds $12 \mathrm{c}$ active against $S$. aureus, B. subtilis, and P. aeruginosa at MIC $30 \mu \mathrm{g} / \mathrm{ml} ; 12 \mathrm{f}$ active against $S$. aureus and B. subtilis at MIC $30 \mu \mathrm{g} / \mathrm{ml} ; 12 \mathrm{~g}$ active against $S$. aureus and B. subtilis at MIC $30 \mu \mathrm{g} / \mathrm{ml}$; and $12 \mathrm{~d}$ and $12 \mathrm{~h}$ active against $S$. aureus, B. subtilis, and P. aeruginosa at MIC $50 \mu \mathrm{g} / \mathrm{ml}$, respectively, when compared with standard drug ciprofloxacin. Similarly, compound $12 \mathrm{~b}$ was active against $A$. niger and C. albicans, respectively, at MIC $30 \mu \mathrm{g} / \mathrm{ml}$ when compared with standard drug fluconazole. The rest compounds $12 \mathrm{a}, 12 \mathrm{~d}, 12 \mathrm{e}$, and $12 \mathrm{~h}$ were active against $C$. albicans, respectively, at MIC $30 \mu \mathrm{g} / \mathrm{ml}$ and $12 \mathrm{c}$, $12 \mathrm{f}$, and $12 \mathrm{~g}$ against $C$. albicans, respectively, at MIC $50 \mu \mathrm{g} / \mathrm{ml}$ when compared with standard fluconazole. These molecules can potentially serve as useful "lead" compounds for further development.

\section{AUTHOR'S CONTRIBUTION}

Dr. Lakhwinder Sing and Anuja Chopra make contributions to the conception, design, and implementation of the research to the analysis of the results and to the writing of the manuscript. Dr. Lakhwinder Singh helped to supervise the work and gave final approval of the written manuscript. Dr. NeelimaDhingra and RichaDhingra work on the molecular docking studies.

\section{ACKNOWLEDGEMENT}

I am thankful to the G. H. G. Khalsa College of Pharmacy, Gurusar Sudhar, Ludhiana, for encouraging us and providing the necessary facilities for carrying out this research work. I gratefully acknowledge the Chairperson, University Institute of Pharmaceutical Sciences and UGC Center of Advanced Study (UGC-CAS) in Pharmaceutical Sciences, Punjab University, Chandigarh, for their support by extending their laboratory facilities. I am thankful Dr. NeelimaDhigra and Ms. Richa for contributing completely for carrying out the docking studies. I am thankful to the CGC College of Engg., Landran, Mohali, for their cooperation and providing us a healthy atmosphere. I am thankful to the RIC Department of IK Gujral PTU, Kapurthala, for providing me the opportunity to pursue my PhD. I gratefully acknowledge IMTECH, Chandigarh, for providing us various bacterial and fungal strains.

\section{CONFLICTS OF INTERESTS}

All authors have none to declare.

\section{REFERENCES}

1. Singh B, Sharma V, Singh G, Kumar R, Arora S, Ishar MP, et al. Synthesis and in vitro cytotoxic activity of chromenopyridones. Int $\mathrm{J}$ Med Chem 2013;2013:984329.
2. Valdameri G, Genoux-Bastide E, Peres B, Gauthier C, Guitton J, Terreux R, et al. Substituted chromones as highly potent nontoxic inhibitors, specific for the breast cancer resistance protein. J Med Chem 2012;55:966-70.

3. Gaspar A, Silva T, Yáñez M, Vina D, Orallo F, Ortuso F, et al. Chromone, a privileged scaffold for the development of monoamine oxidase inhibitors. J Med Chem 2011:54:5165-73.

4. Ramos FA, Takaishi Y, Shirotori M, Kawaguchi Y, Tsuchiya K, Shibata $\mathrm{H}$, et al. Antibacterial and antioxidant activities of quercetin oxidation products from yellow onion (Allium cepa) skin. J Agric Food Chem 2006;54:3551-7.

5. Goel R, Sharma V, Budhiraja A, Ishar MP. Synthesis and evaluation of novel 3a,9a-dihydro-1-ethoxycarbonyl-1-cyclopenteno[5,4-b]benzopyran-4ones as antifungal agents. Bioorg Med Chem Lett 2012;22:4665-7.

6. Kuroda M, Uchida S, Watanabe K, Mimaki Y. Chromones from the tubers of Eranthis cilicica and their antioxidant activity. Phytochemistry 2009; 70:288-93.

7. Brinkworth RI, Stoermer MJ, Fairlie DP. Flavones are inhibitors of HIV-1 proteinase. Biochem Biophys Res Commun 1992;188:631-7.

8. Sandhya B, Vinod M, Lohitha P, Ashwini T, Shravani A. Synthesis, characterization and pharmacological activities of coumarin derivatives. Int J Chem Pharm Sci 2010;1:16-25.

9. Kumar PB, Yerrabelly H, Yerrabelly JR. Synthesis and anti-microbial/ anti-malarial activity of a new class of chromone-dihydroquinazolinone hybrid heterocycles. ARKIVOC 2018;3:212-8.

10. Galal SA, El-All AS, Abdallah MM, El-Diwani HI. Synthesis of potent antitumor and antiviral benzofuran derivatives. Bioorg Med Chem Lett 2009; 19:2420-4

11. Hanna AT, Ewies EF, Wageeh SE. Synthesis of chromones and their applications during the last ten years. Int J Res Pharm Chem 2014;4:1046-85.

12. Serge K, Hubert GT, Janine B, Pierre B, Marie M. Synthesis and evaluate the anti-allergic and antihistamine activity of benzyl-3chromones (homo-isoflavones). Eur J Med Chem 1989;24:541-6.

13. Hatzade K, Taile V, Gaidhane P, Ingle V. Synthesis, structural determination, and biological activity of new 7-hydroxy-3-pyrazolyl4H-chromen- 4-ones and their o-beta-D-glucosides. Turk J Chem 2010;34:241-54.

14. Sashidhara KV, Kumar A, Kumar M, Sarkar J, Sinha S. Synthesis and in vitro evaluation of novel coumarin-chalcone hybrids as potential anticancer agents. Bioorg Med Chem Lett 2010;20:7205-11.

15. Kawase M, Tanaka T, Kan H, Tani S, Nakashima H, Sakagami H, et al. Biological activity of 3-formylchromones and related compounds. In vivo 2007;21:829-34.

16. Ali TE, Magdy AI, Eman ME, Zeinab ME. Proceedings in $16^{\text {th }}$ International Electronic Conference on Synthetic Organic Chemistry. Egypt; 2012. p. 1-15.

17. Mazimba O. Antimicrobial activities of heterocycles derived from thienylchalcones. J King Saudi Univ Sci 2015;15:27-48.

18. Singh G, Sharma S. Synthesis and antimicrobial activity of thiosemicarbazone induced hydrazone of 2-Anilino-3-formylchromone. J Chem Pharm Res 2015;7:599-605.

19. Singh G, Singh R, Girdhar NK, Ishar MP. A versatile route to 2-alkyl-/ aryl-amino-3-formyl- and hetero-annelated-chromones, through a facile nucleophilic substitution at $\mathrm{C} 2$ in 2-(N-methylanilino)-3formylchromones. Tetrahedron 2002;58:2471-80.

20. Singh G, Singh G, Ishar MP. Pericyclic Transformations at the periphery of chromen-4-one ( $=4 H$-1-benzopyran-4-one): An unusual preference for a 1,5-shift of allylic moieties over the ene reaction. Synlett 2003;86:256-8.

21. Singh G, Singh L, Ishar MP. 2-(N-Methylanilino)-3-formylchromone- a versatile synthon for incorporation of chromone moiety in a variety of heterocyclic systems and macrocycles through reactions with bifunctional nucleophiles. Tetrahedron 2002;58:7883-90.

22. Ishar MP, Singh G, Singh S, Sreenivasan KK, Singh G. Design, synthesis, and evaluation of novel 6-chloro-/fluorochromone derivatives as potential topoisomerase inhibitor anticancer agents. Bioorg Med Chem Lett 2006;16:1366-70.

23. Ibrahim SS, Allimony HA, Abdel-Halim AM, Ibrahim AM. Synthesis and reactions of 8-allylchromone-3-carboxaldehyde. ARKIVOC 2009; 14:28-38.

24. Ahmed B, Samad A, Hasan M. Molecular modelling studies, synthesis and antimicrobial screening of some novel sulphonamide quinazolin4(3h)-one fused derivatives. Int J Pharm Pharm Sci 2014;6:312-7.

25. Ramya TS, Sathyanathan T, Kumar PD, Chowdhari M. Docking studies on synthesized quinazoline compounds on androgen receptor. Int J 
Pharm Ind Res 2011;1:266-9

26. Kumar HK, Parmeshwar H. Synthesis, molecular docking and antibacterial evaluation of some novel N-4 piperidinyl derivatives of sparfloxacin. Asian J Pharm Clin Res 2018;11:415-21

27. Ramanjaneyulu K, Bindhu JH, Naaz TU, Prasad VV, Satya BL.
Synthesis, antioxidant, antibacterial and cytotoxic activity of novel chromone derivatives. Pharm Chem 2017;9:78-89.

28. Walmik P, Naraboli BS, Swathi B, Ghanti S. Design, synthesis of biologically active heterocycles containing indol-thiazolylthiazolidinone derivatives. Asian J Pharm Clin Res 2017;11:113-7. 